\title{
Liver Fibrosis and Steatosis in Human Immunodeficiency Virus (HIV) Infected Patients
}

\author{
Kemal Fariz Kalista \\ Division of Hepatobiliary, Department of Internal Medicine, Faculty of Medicine, \\ Universitas Indonesia/Dr. Cipto Mangunkusumo General National Hospital, Jakarta
}

Poorly controlled Human Immunodeficiency Virus (HIV) is known as a risk factor for liver fibrosis. HIV is also known as a major factor for acceleration of liver related disease in patient with HIV-Hepatitis C Virus (HCV) coinfection. While the routine of antiretroviral therapy (ART) has resulted in decrease of morbidity and mortality among HIV-infected patients, the leading causes of death were shifting to non-AIDS diseases such as liver-related, cardiovascular-related, and nonAIDS malignancies. ${ }^{1}$ Etiology of liver-related diseases in addition to hepatitis virus is non-alcoholic fatty liver disease (NAFLD). ${ }^{2}$ NAFLD represents a spectrum of liver disease, commencing from a simple steatosis, leading to nonalcoholic steatohepatitis (NASH), fibrosis and cirrhosis. ${ }^{3}$

In European outpatient clinics $(n=125)$, the prevalence of fibrosis and steatosis was $18 \%$ and $55 \%$ respectively in patients with persistent HIV using abdominal ultrasound and Liver Stiffness Measurement (LSM) $(\geq 7.5 \mathrm{kPa}) .{ }^{4}$ In Indonesia, study by Jasirwan et al showed that significant fibrosis was found in $21 \%$ of patients, and significant steatosis was in $20 \%$ of virally suppressed HIV-infected patients with CMV seropositivity. ${ }^{5}$

There are several risk factors leading to fibrosis and steatosis disease in persons with HIV infection, including metabolic syndrome (hypertension, dyslipidemia, increased waist circumference, insulin resistance), hyperuricemia, HIV-related lipodystrophy, combination antiretroviral therapy (cART), gut microbiome and HIV virus itself. ${ }^{2}$ Jasirwan et al found a few risk factors that may contribute to fibrosis and steatosis in virally suppressed HIV-infected patients with CMV seropositivity. Fibrosis and steatosis were measured by transient elastography with controlled attenuation parameter. The risk factor were triglyceride, LDL, total cholesterol, and HDL level. BMI also has a moderate correlation with liver steatosis and together with triglyceride, could predict the liver steatosis value. Other factors such as sex, HOMA-IR, and platelet count could predict the liver fibrosis value. ${ }^{5}$

Mechanisms of glucose metabolism dysregulation and dyslipidemia in HIV-infected individuals are related to activation of innate immune system and insulin resistance. The cART may suppress HIV infection and reduce inflammation. Nevertheless, the use of ART such as zidovudine, stavudine and didanosine was associated with liver steatosis. ${ }^{6}$ ART changes the dyslipidemia pattern, characterized by an increase in TGs and LDL-C, a reduction in HDL-C, and maintenance of insulin resistance. ${ }^{7}$

Cytomegalovirus (CMV) is a double-stranded DNA virus in the herpesvirus family which is the most common viral opportunistic infection and recognized in up to $40 \%$ of patients with advanced HIV disease. Patients with CMV coexisting with HIV might affect the development of an abnormal liver function. ${ }^{8}$

Long periodic asymptomatic CMV-HIV infection might affect the development of abnormal liver function even though the mechanism of CMV-related liver disease is still unclear. In previous reports, CMV proteins (CMV IE1 or IE2) in other tissue work as inducers of the fibrotic process. IE1 and IE2 gene products have the potency to induce $T G F-\beta 1$ activation, which is well-known as a potent fibrotic molecule, and the subsequent potency of the fibrotic phenotype obtained by transfected cells. Several CMV proteins also control the mechanism of cell apoptosis. ${ }^{9}{ }^{10}$ Liver-related disease is one of the leading causes of non-AIDS-related death. Early diagnosis, risk stratify and prompt treatment of liver disease in its early stages are crucial factors to reduce morbidity and mortality in HIV-infected patients.

\section{REFERENCES}

1. Palella FJ, Delaney KM, Moorman AC, Loveless MO, Fuhrer J, Satten GA, et al. Declining morbidity and mortality among patients with advanced human immunodeficiency virus infection. N Engl J Med 1998;338:853-60. 
2. Seth A, Sherman KE. Fatty liver disease in persons with HIV infection. Top Antivir Med 2019;27:75-82.

3. Welzen BJ, Mudrikova T, Idrissi AE, Hoepelman AI, Arends JE. A review of non-alcoholic fatty liver disease in HIV-infected patients: the next big things? Infect Dis Ther 2019:8:33-50

4. Lombardi R, Sambatakou H, Mariolis I, Cokkinos D, Papatheodoridis GV, Tsochatzis EA. Prevalence and predictors of liver steatosis and fibrosis in unselected patients with HIV mono-infection. Dig Liver Dis 2016;48:1471-1477.

5. Jasirwan COM, Aprilia G, Purnamasari D, Yunihastuti E, Gani RA, Wibowo A, et al. Fibrosis and steatosis in virally suppressed HIV-infected patients with cytomegalovirus seropositivity. Indones J Gastroenterol Hepatol Dig Endosc 2021;22:180-7.

6. Perazzo H, Cardoso SW, Yanavich C, Nunes EP, Morata M, Gorni $\mathrm{N}$ et al. Predictive factors associated with liver fibrosis and steatosis by transient elastography in patients with HIV mono-infection under long-term combined antiretroviral therapy. JIAS 2018;21;25201

7. Bischoff J, Gu W, Zander CS, Boesecke C, Wasmuth JC, Bremen KV, et al. Stratifying the risk of NAFLD in patients with HIV under combination antiretroviral therapy (cART).E Clin Med 2021;40:101116.

8. Cheung TW, Teich SA. Cytomegalovirus infection in patients with HIV infection. Mt Sinai J Med 1999;66:113-24.

9. Shimamura M, Murphy-Ullrich JE, Britt WJ. Human cytomegalovirus induces TGF- $\beta 1$ activation in renal tubular epithelial cells after the epithelial-to-mesenchymal transition. PLoS Pathog 2010;6:e1001170.

10. Jeong W Il, Park O, Radaeva S, Gao B. STAT1 inhibits liver fibrosis in mice by inhibiting stellate cell proliferation and stimulating NK cell cytotoxicity. Hepatology 2006;44:1441-5. 